\title{
Endoscopy-assisted vitrectomy vs. vitrectomy alone: comparative study in complex retinal detachment with proliferative vitreoretinopathy
}

\author{
Flavio A. Rezende ${ }^{1}$, Natalia Vila2* ${ }^{2^{*}}$ and Emmanouil Rampakakis ${ }^{3}$
}

\begin{abstract}
Background: Recurrent retinal detachment (RD) is still a widespread event despite the therapeutic options available. Proliferative vitreoretinopoathy (PVR) is one of the main causes of redetachment. Little is known about the use of endoscopy-assisted vitrectomy (E-PPV) in complex recurrent RD with PVR. The purpose of this study was to identify the potential advantages of E-PPV in complex RD with PVR compared with pars plana vitrectomy (PPV) alone.

Methods: Single-center, retrospective, observational, descriptive study. The medical records of 293 patients were reviewed. Patients who underwent PPV for complex rhegmatogenous RD and associated PVR between 2009 and 2017 were included. Patients with diabetic tractional RD, trauma, uveitis or detachment postendophthalmitis were excluded. After 2013, an endoscopic visualization system was used in a nonrandomized fashion at the surgeon's discretion. Outcome measures (reattachment rate, number of surgeries, lens status, PVR stage, intraocular pressure, phthisis rate) were compared between the E-PPV and PPV-only groups with independent samples t-tests (continuous variables) and Fisher's exact test (categorical variables), as well as time-adjusted analyses. Postoperative time to retinal redetachment was assessed with Kaplan-Meier survival analysis.
\end{abstract}

Results: One hundred one eyes from 100 patients met the inclusion criteria. The mean participant age was 63.3 years old (95\% Cl 60.4-66.1 years), without a significant difference between groups. E-PPV was performed in 36.6\% ( $n=37)$ of eyes, and $63.4 \%(n=64)$ underwent PPV only. The mean follow-up was significantly longer in the PPV-only group (31.9 vs. 21.1 months; $p=0.021$ ). Upon adjustment for follow-up duration, the mean number of surgeries was significantly lower in the PPV-only group (2.6 vs. 4.3 number of surgeries; $p<0.001$ ) than in the E-PPV group. A significantly higher risk for redetachment was observed in the PPV-only group (HR [95\% Cl] 4.1 [1.4-11.8]) than in the E-PPV group $(p=0.037)$. The evolution to phthisis was 7\% $(n=4)$ in the PPV-only group and $2.7 \%(n=1)$ in the E-PPV group $(p>0.05)$.

Conclusions: Compared to PPV alone, endoscopy-assisted vitrectomy seems to be advantageous in achieving better reattachment rates in complex RD with advanced PVR. Endoscopic visualization allows a thorough examination and extensive anterior PVR and vitreous base dissection.

*Correspondence: natalia.vila@rlbuht.nhs.uk

${ }^{2}$ St Paul's Eye Unit, Royal Liverpool University Hospital NHS Trust, Prescot

St, Liverpool L7 8XP, UK

Full list of author information is available at the end of the article

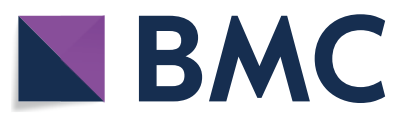

(c) The Author(s) 2020. This article is licensed under a Creative Commons Attribution 4.0 International License, which permits use, sharing, adaptation, distribution and reproduction in any medium or format, as long as you give appropriate credit to the original author(s) and the source, provide a link to the Creative Commons licence, and indicate if changes were made. The images or other third party material in this article are included in the article's Creative Commons licence, unless indicated otherwise in a credit line to the material. If material is not included in the article's Creative Commons licence and your intended use is not permitted by statutory regulation or exceeds the permitted use, you will need to obtain permission directly from the copyright holder. To view a copy of this licence, visit http://creativeco mmons.org/licenses/by/4.0/. The Creative Commons Public Domain Dedication waiver (http://creativecommons.org/publicdomain/ zero/1.0/) applies to the data made available in this article, unless otherwise stated in a credit line to the data. 
Keywords: Endoscopy-assisted vitrectomy, Endoscopy-guided vitrectomy, Endoscopic vitrectomy, Proliferative vitreoretinopathy, Retinal detachment

\section{Background}

Recurrent rhegmatogenous retinal detachment (RRD) after surgical repair remains a relatively widespread event despite the therapeutic options currently available. Proliferative vitreoretinopoathy (PVR) is one of the main causes of redetachment and occurs in $8-10 \%$ of patients undergoing primary repair [1-4]. The search for better anatomical outcomes has been investigated by comparing or combining surgical techniques [5, 6]. Little is known in the literature about the addition of endoscopy when performing a vitrectomy in recurrent retinal detachment, especially in complex detachments with PVR [7-12].

Vitreoretinal surgery is undergoing an era of technification. Endoscopic vitrectomy has changed over the past years, and currently, it can be integrated into digitally assisted visualization systems to facilitate learning curves and ease of use [13]. There is still a need to improve surgical outcomes in complex cases, and endoscopy-assisted vitrectomy could facilitate and address anterior pathology differently in these patients.

The aim of the current study was to compare the efficacy of endoscopy-assisted pars plana vitrectomy (E-PPV) vs. pars plana vitrectomy (PPV) alone in complex RRD with advanced PVR.

\section{Methods}

This was a single-center, retrospective, observational, descriptive study conducted in accordance with the tenets of the Declaration of Helsinki. The protocol was approved by the local Institutional Review Board. Informed consent was waived due to the retrospective nature of the study. Medical records of the Department of Ophthalmology at Maisonneuve-Rosemont Hospital (CIUSS de l'est d'Ile de Montréal, University of Montreal, Montreal, Quebec, Canada) were screened for patients who underwent PPV for RRD associated with PVR between July 2nd, 2009 and January 31st, 2017. A total of 293 surgical reports were reviewed, and patients meeting the following criteria were included in the study: 18 years of age or more, RRD associated with advanced PVR and a minimum follow-up of 6 months post-PPV. The classification of PVR used was Machemer's proposal [14], and we included the following categories: (PVR-B) wrinkling of the inner retina surface, retinal stiffness, and/or retinal breaks with rolled edges; (PVR-C1) starfolds posterior to the vitreous base (VB); (PVR-C2) confluent starfolds posterior to the VB; (PVR-C3) proliferation under the retina, annular strand near the disc, linear strands or moth-eaten-appearing sheets (anterior or posterior); (PVR-C4) retina contraction inwards at the posterior edge of the VB, with central displacement of the retina, peripheral retina stretched or posterior retina in radial folds; (PVR C-5) anterior contraction on the retina at the vitreous base, presence of ciliary body (CB) detachment and epiciliary membrane, or iris retraction. The RRD with PVR included in this study, which we referred to as "advanced PVR", had PVR greater than PVR-A (PVR-B and/or C). Participants with diabetic tractional detachment, an exudative component, a history of trauma (blunt trauma or open globe injury), uveitis or endophthalmitis were excluded. After 2013, an endoscopic visualization system (E2 MicroProbe ${ }^{\mathrm{TM}}$; EndoOptiks, Little Silver, USA) was used in a nonrandomized fashion at the surgeon's discretion. Eligible patients were divided into two groups: (1) the endoscopy-assisted PPV (E-PPV) group, who underwent surgery that combined endoscopic visualization with wide-field visualization, and (2) the PPV-alone group, for whom endoscopy was never used during any of the surgical interventions during follow-up. Patients who underwent more than one surgery and for whom endoscopy was used in at least one surgical procedure during follow-up were included in the E-PPV group.

A minimum of four ocular examinations were performed: prior to surgery, postoperatively (day 1), approximately 1 month after surgery (4 to 6 weeks) and at least 6 months after surgery. All examinations included best corrected visual acuity (BCVA), intraocular pressure (IOP) measurement with applanation tonometry $(\mathrm{mmHg})$, slit lamp biomicroscopy and indirect ophthalmoscopy. The primary outcome was the rate of retinal reattachment, and secondary outcomes were the number of surgeries, lens status, PVR stage, IOP and phthisis rate.

Surgical procedures were performed using 23-gauge or 25 -gauge (CONSTELLATION ${ }^{\circledR}$ Vision System) platforms under a microscope with a noncontact wide-field visualization system. In the E-PPV group, a 23-gauge or a 20-gauge probe was used in combination with the standard vitrectomy setup. Endoscopy was performed through a 23-gauge trocar or a 20-gauge sclerotomy. Data extracted from the surgical reports were lens status at the end of the procedure, use of perfluorocarbon liquid, membrane peeling (ERM, ILM, PVR), degrees of retinectomy, laser use, retinectomy extension, anterior retinectomy flap trimming, tamponade and ciliary body (CB) status (when available). 


\section{Statistics}

Statistical analysis was performed using SPSS software version 24 (Armonk, NY: IBM Corp.). Summary statistics were produced for all study variables, consisting of the mean and 95\% confidence intervals (95\% CI) for continuous variables, as well as frequency distributions for categorical variables. Patient characteristics and preoperative and intraoperative ocular characteristics in the E-PPV and PPV-only groups were compared using the independent samples t-test for continuous variables and the Chi-squared or Fisher's exact test, as appropriate, for categorical variables. Comparison of the E-PPV and PPV-only groups in terms of postoperative outcomes was conducted using logistic regression (phthisis), generalized linear models (BCVA, IOP), and Poisson regression (number of surgeries) adjusting for duration of follow-up. Postoperative time to retinal redetachment was assessed with Kaplan-Meier survival analysis, and the Greenwood method was used to calculate the $95 \% \mathrm{CI}$ of the hazard ratio.

\section{Results}

The study included 101 eyes from 100 patients who underwent PPV for RRD associated with PVR. Endoscopy-assisted PPV was performed in $36.6 \%(n=37)$ of the eyes, and the remaining $63.4 \%(n=64)$ underwent PPV only. The demographic characteristics did not show statistically significant differences between groups (Table 1), although the mean age was numerically lower in the PPV-only group than in the E-PPV group (61.9 vs. 65.6). Preoperative ocular characteristics were similar between the groups (Table 2), with the exception of the underlying diagnosis (RRD and PVR, recurrent $R D$, recurrent $R D$ under oil, recurrent RD postoil removal), which was significantly different $(\mathrm{p}=0.016)$ between groups.

\section{Table 1 Patient characteristics}

\begin{tabular}{lccc}
\hline & PPV-only & E-PPV & p value \\
\hline Number of eyes, n (\%) & $64(63.4)$ & $37(36.6)$ & N/A \\
$\begin{array}{l}\text { Number of patients, n (\%) } \\
\text { Gender, n (\%) }\end{array}$ & $64(64.0)$ & $36(36.0)$ & N/A \\
Male & $41(64.1)$ & $25(69.4)$ & 0.663 \\
Female & $23(35.9)$ & $11(30.6)$ & \\
Age at surgery (years) & & & \\
Mean (95\% Cl) & $61.9(57.8-66.1)$ & $65.6(62.9-68.4)$ & 0.217 \\
Eye, n (\%) & & & 0.834 \\
OD & $37(57.8)$ & $23(62.2)$ & \\
OS & $27(42.2)$ & $14(37.8)$ &
\end{tabular}

N/A not applicable, PPV pars plana vitrectomy, E-PPV endoscopy-assisted PPV

a Proportions based on the total number of patients included

b Proportions based on the total number of eyes included
Table 2 Preoperative ocular characteristics

\begin{tabular}{lccc}
\hline & PPV-only & E-PPV & p value \\
\hline $\begin{array}{l}\text { Baseline BCVA (LogMAR) } \\
\text { Mean (95\% Cl) }\end{array}$ & $1.59(1.36-1.82)$ & $1.60(1.30-1.91)$ & 0.944 \\
IOP (mmHg) & & & \\
Mean (95\% Cl) & $12.3(10.5-14.0)$ & $11.3(9.3-13.3)$ & 0.474 \\
Lens status, n (\%) & & & \\
Phakic & $18(32.7)$ & $2(5.6)$ & 0.002 \\
Pseudophakic & $37(67.3)$ & $34(94.4)$ & \\
$\begin{array}{l}\text { Diagnosis, n (\%) } \\
\text { RD + PVR }\end{array}$ & $31(48.4)$ & $11(29.7)$ & 0.016 \\
Recurrent RD & $21(32.8)$ & $8(21.6)$ & \\
Recurrent RD under SO & $7(10.9)$ & $12(32.4)$ & \\
Recurrent RD post-SO & $5(7.8)$ & $6(16.2)$ & \\
$\quad$ removal & & & \\
\hline
\end{tabular}

$P P V$ pars plana vitrectomy, BCVA best corrected visual acuity, E-PPV endoscopyassisted PPV, IOP intraocular pressure, $I O L$ intraocular lens, $R D$ retinal

detachment, PVR proliferative vitreoretinopathy, SO silicone oil

a Proportions based on the total number of eyes included

b Lens status was missing for nine eyes in the PPV only group and one eye in the endoscopy-assisted PPV group

Over a mean (95\% CI) of 31.9 (25.2-38.6) and 21.1 (16.4-25.8) months of follow-up in the PPV-only and E-PPV groups, respectively, the number of surgeries was significantly higher in the E-PPV group, with 4.1 surgeries compared to 2.7 in the PPV-only group $(\mathrm{p}<0.001)$ (Table 4); adjustment for follow-up duration showed similar statistically significant results. Intraoperatively, in the PPV-only group, the 25-gauge vitrectomy platform was predominantly used (94.3\%), while in the E-PPV group, the 25-gauge platform was used in $60 \%$ of cases and the 23 -gauge platform was used in $40 \%(\mathrm{p}<0.001)$ (Table 3$)$. The prevalence of PVR types was similar between the groups, except for PVR-C5, which was higher in E-PPV $(9.4 \%$ vs. $32.4 \% ; \mathrm{p}=0.006)$. The same group also presented a higher percentage of perisilicone oil proliferation (3.1\% vs. $13.5 \% ; \mathrm{p}=0.048)$. Overall, large retinectomies were warranted in $44.5 \%$ of patients undergoing the last surgical procedure. The proportion of retinectomies larger than $270^{\circ}$ was similar in both groups; however, $180^{\circ}$ retinectomies were performed in $37.8 \%$ of the E-PPV group compared to $17.2 \%$ of the PPV-only group, and this difference was statistically significant $(\mathrm{p}=0.031)$ (Table 3). The tamponade used in the last surgical procedure was not significantly different $(p=0.119)$; eyes with silicone oil used as a tamponade did not show differences between groups $(\mathrm{p}=0.315)$ (Table 4). The distribution of intraocular lenses (IOLs) in the posterior capsular bag, IOLs in the anterior chamber, scleral fixated IOLs or aphakia postsurgery was similar between the two groups $(\mathrm{p}=0.276)$. 
Table 3 Intraoperative ocular characteristics

\begin{tabular}{|c|c|c|c|}
\hline & PPV-only & E-PPV & $p$ value \\
\hline \multicolumn{4}{|l|}{ PVR } \\
\hline $\mathrm{B}, \mathrm{n}(\%)^{\mathrm{a}}$ & $26(40.6)$ & 18 (48.6) & 0.533 \\
\hline \multicolumn{4}{|l|}{ C, $n(\%)^{a}$} \\
\hline 1 & $19(29.7)$ & $6(16.2)$ & 0.156 \\
\hline 2 & $15(23.4)$ & $7(18.9)$ & 0.803 \\
\hline 3 & $11(17.2)$ & $6(16.2)$ & $>0.999$ \\
\hline 3-Anterior & $4(6.3)$ & $3(8.1)$ & 0.705 \\
\hline 3-Posterior & $7(10.9)$ & $3(8.1)$ & 0.742 \\
\hline 4 & $6(9.4)$ & $3(8.1)$ & $>0.999$ \\
\hline 5 & $6(9.4)$ & $12(32.4)$ & 0.006 \\
\hline \multicolumn{4}{|c|}{ Perisilicone proliferation } \\
\hline $\mathrm{n}(\%)^{\mathrm{a}}$ & (3.1) & $(13.5)$ & 0.048 \\
\hline \multicolumn{4}{|l|}{ Retinectomy } \\
\hline $180^{\circ}, \mathrm{n}(\%)^{\mathrm{a}}$ & $11(17.2)$ & $14(37.8)$ & 0.031 \\
\hline $270^{\circ}, \mathrm{n}(\%)^{\mathrm{a}}$ & $4(6.3)$ & $3(8.1)$ & 0.705 \\
\hline $360^{\circ}, \mathrm{n}(\%)^{\mathrm{a}}$ & $7(10.9)$ & $6(16.2)$ & 0.541 \\
\hline \multicolumn{4}{|l|}{ Tamponade ${ }^{b}$} \\
\hline Air, n (\%) & $1(1.9)$ & $3(8.3)$ & 0.119 \\
\hline SF6, n (\%) & $0(0)$ & $2(5.6)$ & \\
\hline $\mathrm{C} 3 \mathrm{~F} 8, \mathrm{n}(\%)^{\mathrm{a}}$ & $14(26.4)$ & $6(16.7)$ & \\
\hline so, n $(\%)^{a, c}$ & $38(71.7)$ & $25(69.4)$ & \\
\hline \multicolumn{4}{|c|}{ PPV (wide-field) } \\
\hline $25 \mathrm{~g}, \mathrm{n}(\%)^{\mathrm{a}}$ & $50(94.3)$ & $21(60.0)$ & $<0.001$ \\
\hline $23 \mathrm{~g}, \mathrm{n}(\%)^{\mathrm{a}}$ & $3(5.7)$ & $14(40.0)$ & \\
\hline OR (95\% Cl) & $11.1(2.9-43.5)$ & Ref. & \\
\hline
\end{tabular}

PPV pars plana vitrectomy, E-PPV endoscopy-assisted PPV, PVR proliferative vitreoretinopathy, Ref reference category, SO silicone oil

a Proportions based on the total number of eyes included

b Tamponade information was missing for eleven eyes in the PPV only group and one eye in the endoscopy-assisted PPV group

c An additional 4 patients in the PPV-only group and 8 patients in the E-PPV group had used SO in a previous surgery

At the final follow-up, the reattachment rate was $78.9 \%$ in the PPV-only group and $94.6 \%$ in the E-PPV group $(\mathrm{p}=0.037)$ (Fig. 1); in a sensitivity analysis excluding the group of patients with silicone oil-filled eyes, the reattachment rate at the final follow-up in the PPV-only and E-PPV groups was $87.9 \%$ and $100 \%$, respectively. The Kaplan-Meier estimated time to redetachment was also significantly shorter in the PPV-only group, corresponding to a fourfold increase in the hazard rate (HR [95\% CI] 4.1 [1.4-11.8]) (Fig. 2). Phthisis was $7 \%(n=4)$ in the PPV-only group and $2.7 \%(n=1)$ in the E-PPV group but did not reach statistical significance in either unadjusted analysis (OR [95\% CI] 2.7 [0.3-25.3]) or after adjusting for follow-up duration (OR [95\% CI] 3.0 [0.3-28.4]. No differences were found when comparing final VA or IOP between groups (Table 4).

\section{Discussion}

Our results show that E-PPV may favor reattachment in RRD with advanced PVR. To the best of our knowledge, this is the first study comparing E-PPV vs. PPV alone.

The ability to have an undistorted view of the anterior $\mathrm{VB}$ as well as the retroiridial space and $\mathrm{CB}$, enables the identification of different potential causes of redetachment that would not be detected with a wide-field visualization system during PPV alone and scleral indentation. Our intraoperative results revealed the following findings: undetected anterior traction, identification of anterior hyaloid contractions, contraction of the anterior edge of prior retinectomies, and identification of anterior PVR and CB traction. In addition, the ability to have better access to perform a more peripheral retinectomy, to peel multiple layers of the anterior hyaloid and the anterior ring of contraction at the Salzmann ligament area, and identify the need for capsular bag complex removal are also contributory factors that could explain the observed differences in reattachment rate (Additional file 1: Video S1). Nonetheless, the ability to identify the severity of anterior PVR at the CB region by examining the clinical appearance of the ciliary processes could have prognostic value.

We believe that the identification of these findings may have contributed to better anatomical results when E-PPV was performed. Boucher and Kuhn [8] reported the outcomes of 67 eyes after failed postprimary repair, and they identified the following findings with endoscopy: (1) persistent radial and/or circumferential vitreous adherences of the anterior vitreous base to the ciliary body, zonules, posterior lens capsule and iris; (2) persistent circumferential adherences inside the posterior part of the VB; (3) persistent anterior and posterior adherences; (4) reproliferation at the sclerotomy site; (5) CB detachment; (6) retinal break; (7) anterior neovascularization; and (9) subretinal proliferation. They concluded that the unique information provided by endoscopy correlated with experimental and pathological studies about the pathogenesis of anterior PVR. Yokohama et al. [12] evaluated the clinical outcomes for E-PPV in uncomplicated RRD, and the primary success rate was $98.4 \%$. In our study, patients who underwent E-PPV showed a 94.6\% reattachment rate, similar to uncomplicated RRD repair results, despite being a series of complex RRD with advanced PVR. Our cohort of patients differed from Boucher's and Yokohama's patients because they were recurrent RD with advanced PVR. Although our 
Table 4 Postoperative ocular characteristics and outcomes at last follow up

\begin{tabular}{|c|c|c|c|c|c|c|}
\hline & \multicolumn{3}{|c|}{ Unadjusted analysis } & \multicolumn{3}{|c|}{ Time-adjusted analysis ${ }^{a}$} \\
\hline & PPV-only & E-PPV & $p$ value & PPV-only & E-PPV & $p$ value \\
\hline \multicolumn{7}{|l|}{ BCVA (logMAR) } \\
\hline Mean $(95 \%$ Cl) & $1.42(1.17-1.68)$ & $1.34(1.09-1.60)$ & 0.201 & $1.44(1.21-1.68)$ & $1.32(1.02-1.61)$ & 0.518 \\
\hline \multicolumn{7}{|l|}{$\mathrm{IOP}(\mathrm{mmHg})$} \\
\hline Mean $(95 \%$ Cl) & $14.5(12.6-16.5)$ & $13.6(11.2-16.1)$ & 0.543 & $14.5(12.5-16.5)$ & $13.7(11.3-16.1)$ & 0.613 \\
\hline \multicolumn{7}{|l|}{ Phthisis } \\
\hline n (\%) & $4(7)$ & $1(2.7)$ & & $\mathrm{N} / \mathrm{A}$ & $\mathrm{N} / \mathrm{A}$ & \\
\hline $\mathrm{OR}(95 \% \mathrm{Cl})$ & $2.7(0.3-25.3)$ & Ref. & 0.362 & $3.0(0.3-28.4)$ & Ref. & 0.342 \\
\hline \multicolumn{7}{|c|}{ Follow-up (months) } \\
\hline Mean $(95 \%$ Cl) & $31.9(25.2-38.6)$ & $21.1(16.4-25.8)$ & 0.021 & $\mathrm{~N} / \mathrm{A}$ & $\mathrm{N} / \mathrm{A}$ & $\mathrm{N} / \mathrm{A}$ \\
\hline \multicolumn{7}{|c|}{ Number of surgeries } \\
\hline Mean $(95 \%$ Cl) & $2.7(2.4-3.1)$ & $4.1(3.5-4.8)$ & $<0.001$ & $2.6(2.2-3.1)$ & $4.3(3.6-5.0)$ & $<0.001$ \\
\hline \multicolumn{7}{|c|}{ Silicone oil removed } \\
\hline$n(\%)^{b}$ & $18(42.9)$ & $18(54.4)$ & 0.315 & $\mathrm{~N} / \mathrm{A}$ & $\mathrm{N} / \mathrm{A}$ & 0.099 \\
\hline OR $(95 \% \mathrm{Cl})$ & $0.6(0.2-1.6)$ & Ref. & & $0.4(0.2-1.2)$ & Ref. & \\
\hline
\end{tabular}

PPV pars plana vitrectomy, BCVA best corrected visual acuity, E-PPV endoscopy-assisted PPV, IOP intraocular pressure, IOL intraocular lens, N/A not applicable, Ref reference category

a Adjusted for duration of follow-up

b Proportions based on eyes with silicon oil as tamponade $\left(n_{P P V-o n l y}=42 ; n_{E-P P V}=33\right)$

intraoperative findings were mostly related to anterior pathology, we also identified contraction of the edge of prior retinectomies in patients with PVR-B; furthermore, subretinal PVR was easily detected with endoscopy. These findings could explain why in cases of posterior PVR the reattachment rate was higher in the E-PPV group. In addition, as seen in one of our previous studies [15], during silicone oil removal the most common intraoperative endoscopy finding was contraction of previous retinectomy in patients with anterior and/or posterior PVR.

Effective drug management for PVR prevention or treatment remains an unmet need. The mainstay method for the management of PVR is surgery, but several studies have demonstrated that the interplay between cytokines, growth factors, matrix proteins and different cell types leads to the formation of pre, intra, and subretinal membranes. Different drugs and drug-delivery systems have been tested for the treatment of PVR. Pharmacologic agents such as anti-inflammatory drugs, antineoplasic/ antiproliferative agents, antigrowth factors and antioxidants are currently being studied. The prevention of PVR will be a very important advancement in the prevention of redetachment; however, once the retina is redetached in PVR, it is imperative to treat this condition. E-PPV can help better identify and treat anterior PVR and potentially offers better anatomic results than PPV alone.
Limitations of this study are its retrospective nature, as well as the nonrandomized use of endoscopy. As such, due to the presence of potential confounders, the study findings should be interpreted as associations, and randomized prospective studies are necessary to draw causal inferences. There is a possible selection bias in the E-PPV group towards more complex RRDs, particularly in the early years of endoscopy adaptation, when it was applied when anterior PVR was clinically identified after failure in recurrent RD; in more recent years, endoscopy has been used routinely in combination with the 23-gauge platform in almost all pseudophakic recurrent redetachments, irrespective of the presence of clinically identifiable anterior PVR. This may explain the statistically higher presence of $\mathrm{C} 5$ and the higher number of surgical procedures used in the E-PPV group. The follow-up in the PPV-only group was longer than that in the E-PPV group because endoscopy only became available in 2013 at our institution. However, despite the different followup periods in each group, all patients were followed for a minimum of 6 months after the last surgery, which is an accepted standard follow-up time among vitreoretinal surgeons regarding postoperative retinal reattachment stability. In addition, a time-adjusted analysis was conducted in an effort to account for this difference. Finally, it is possible that some differences in postoperative outcomes were not identified as statistically significant due 


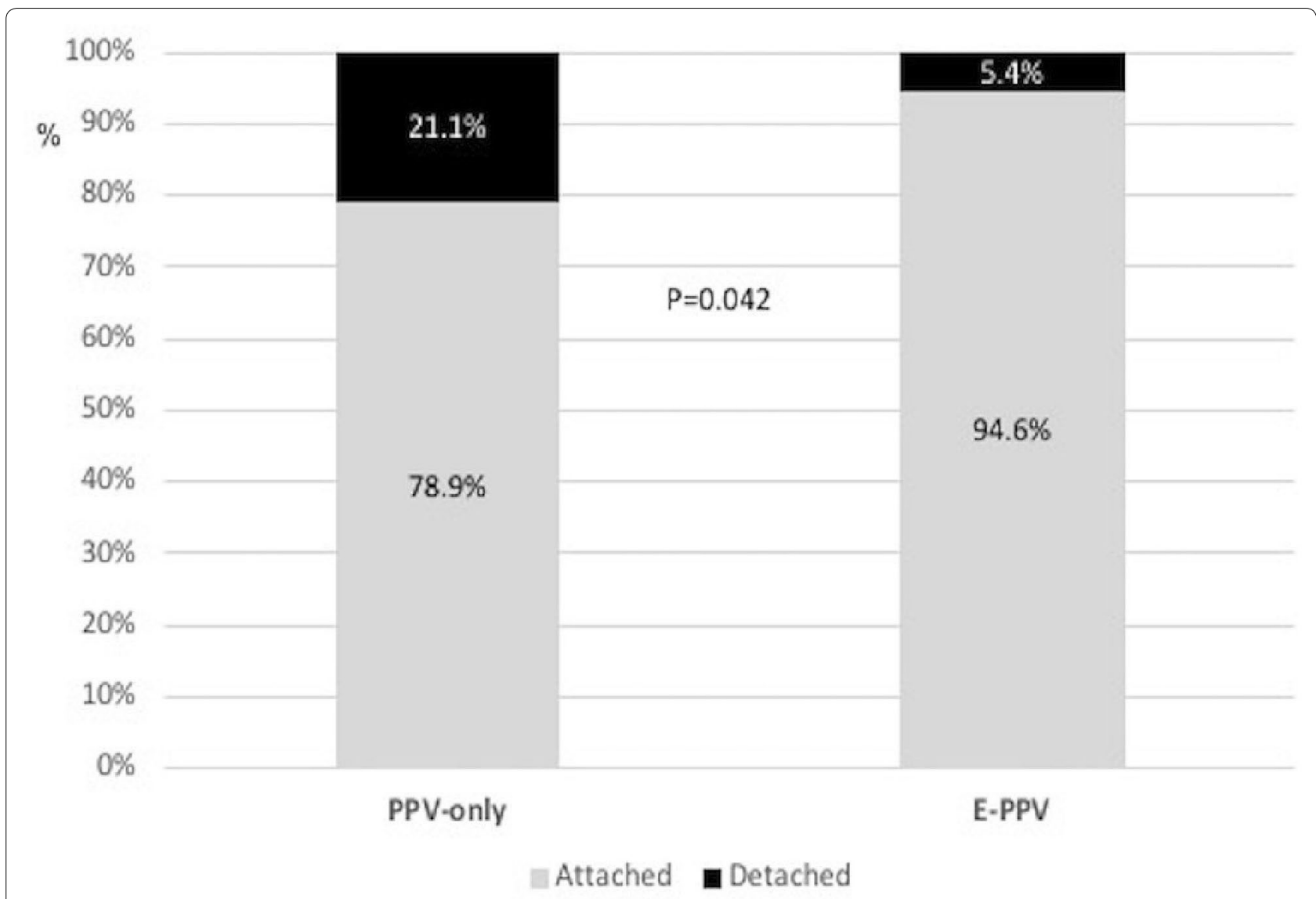

\section{PPV: pars plana vitrectomy; E-PPV: endoscopy assisted pars plana vitrectomy.}

Fig. 1 Retina final status. PPV pars plana vitrectomy, E-PPV endoscopy assisted pars plana vitrectomy

to a lack of statistical power; therefore, in interpreting statistically nonsignificant results, emphasis should be placed on clinical meaningfulness.

We included eyes with a minimum follow-up of 6 months after the last surgery regardless of the type of tamponade used in the last surgical procedure. The distribution of silicone oil-filled eyes was similar in both groups. Silicone oil was the long-term tamponade of choice in both groups in the following scenarios: monocular status, hypotony or patient choice. When excluding the group of patients with silicone oil-filled eyes, the attachment rate at final follow-up in the E-PPV vs. PPVonly groups was $100 \%$ vs. $87.9 \%$. Although the study was not powered to detect this difference as statistically significant, the difference is clinically meaningful and merits further investigation in larger studies.
Unfortunately, few vitreoretinal surgeons have experience with E-PPV. This may be due to several factors, including a lack of image quality in two dimensions, difficulty in reaching the far periphery with current instrumentation and the small number of fellowship programs teaching this technique. However, recent technological improvements, such as the introduction of digitally enhanced visualization systems that increase the image resolution on a $4 \mathrm{~K}$ monitor and allow simultaneous viewing of both endoscopic and wide-field 3D images, should ease the challenges of learning E-PPV. This advancement, coupled with the positive results of the present study highlighting the potentially improved results using E-PPV in complex RRD, could reignite the interest of surgeons and industry investment in better technologies to address the anterior $\mathrm{VB}$ and retroiridial space. 


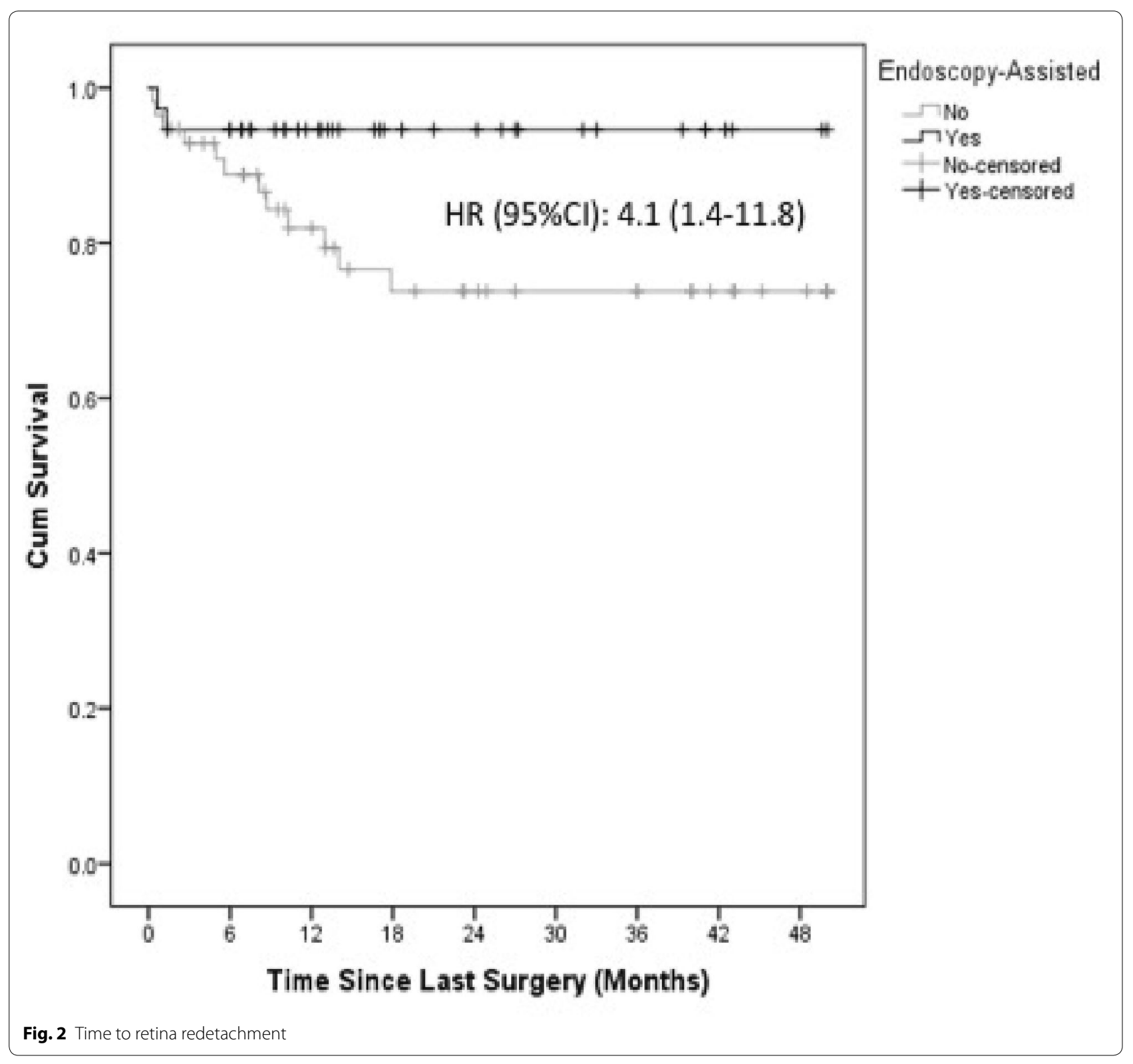

\section{Conclusions}

In this retrospective study, compared to PPV alone, endoscopy-assisted PPV was shown to be advantageous in achieving better reattachment rates in complex RRD with advanced PVR. Endoscopic visualization allowed a thorough examination and extensive anterior PVR and vitreous base dissection. Only one patient evolved to phthisis in the endoscopy group, despite a mean of 4 surgeries needed to achieve anatomical success.

\section{Supplementary information}

Supplementary information accompanies this paper at https://doi. org/10.1186/s40942-020-00238-9.

Additional file 1: Video S1. Endoscopic intraoperative findings.

\section{Abbreviations}

BCVA: Best corrected visual acuity; CB: Ciliary body; Cl: Confidence intervals; E-PPV: Endoscopy-assisted pars plana vitrectomy; ERM: Epiretinal membrane; ILM: Internal limiting membrane; IOL: Intraocular lens; IOP: Intraocular pressure; PPV: Pars plana vitrectomy; PVR: Proliferative vitreoretinopoathy; RD: 
Retinal detachment; RRD: Rhegmatogenous retinal detachment; VA: Visual acuity; VB: Vitreous base.

\section{Acknowledgements}

We greatly appreciate Aouatef Benlemmouden for the technical assistance.

\section{Authors' contributions}

FR: conception, to have approved the submitted version and to have agreed both to be personally accountable for the author's own contributions and to ensure that questions related to the accuracy or integrity of any part of the work, even ones in which the author was not personally involved, are appropriately investigated, resolved, and the resolution documented in the literature. NV: conception, acquisition, analysis, interpretation of data, to have drafted the work, to have approved the submitted version and to have agreed both to be personally accountable for the author's own contributions and to ensure that questions related to the accuracy or integrity of any part of the work, even ones in which the author was not personally involved are appropriately investigated, resolved, and the resolution documented in the literature. ER: analysis and interpretation of data, to have approved the submitted version and to have agreed both to be personally accountable for the author's own contributions and to ensure that questions related to the accuracy or integrity of any part of the work, even ones in which the author was not personally involved, are appropriately investigated, resolved, and the resolution documented in the literature. All authors read and approved the final manuscript.

\section{Funding}

The authors received no financial support for the research, authorship and/or publication of this article.

\section{Availability of data and materials}

The datasets used and/or analysed during the current study are available from the corresponding author on reasonable request.

\section{Ethics approval and consent to participate}

This study is an institutional retrospective case series and was conducted in accordance with the Declaration of Helsinki. The protocol was approved by the local Institutional Review Board. Informed consent was waived due to the retrospective nature of the study.

\section{Consent for publication}

BMC Consent form is signed by 5 patients. The video included as supplemental material includes images form the surgical videos of the aforementioned patients.

\section{Competing interests}

The authors declare that they have no competing interests.

\section{Author details}

1 Department of Ophthalmology (Centre Universitaire d'Ophtalmologie), Maisonneuve Rosemont Hospital, CIUSS de l'est d'lle de Montréal, Montreal, QC, Canada. ${ }^{2}$ St Paul's Eye Unit, Royal Liverpool University Hospital NHS Trust, Prescot St, Liverpool L7 8XP, UK. ${ }^{3}$ Medical Affairs, JSS Medical Research, Montreal, Canada.

Received: 5 March 2020 Accepted: 22 July 2020

Published online: 29 July 2020

\section{References}

1. Leaver PK. Proliferative vitreoretinopathy. Br J Ophthalmol. 1995;79(10):871-2

2. Quiram PA, Gonzales CR, Hu W, et al. Outcomes of vitrectomy with inferior retinectomy in patients with recurrent rhegmatogenous retinal detachments and proliferative vitreoretinopathy. Ophthalmology. 2006:113(11):2041-7.

3. Joeres S, Kirchhof B, Joussen AM. PVR as a complication of rhegmatogeneous retinal detachment: a solved problem? Br J Ophthalmol. 2006;90(6):796-7.

4. Pastor JC, Rojas J, Pastor-Idoate S, Di Lauro S, Gonzalez-Buendia L, Delgado-Tirado S. Proliferative vitreoretinopathy: a new concept of disease pathogenesis and practical consequences. Prog Retin Eye Res. 2016:51:125-55.

5. Khan MA, Brady CJ, Kaiser RS. Clinical management of proliferative vitreoretinopathy: an update. Retina. 2015;35(2):165-75.

6. Charteris DG, Sethi CS, Lewis GP, Fisher SK. Proliferative vitreoretinopathydevelopments in adjunctive treatment and retinal pathology. Eye. 2002;16(4):369-74.

7. Lee GD, Goldberg RA, Heier JS. Endoscopy-assisted vitrectomy and membrane dissection of anterior proliferative vitreoretinopathy for chronic hypotony after previous retinal detachment repair. Retina. 2016;36(6):1058-63.

8. Boscher C, Kuhn F. Endoscopic evaluation and dissection of the anterior vitreous base. Ophthalmic Res. 2015;53:90-9.

9. Sabti KA, Raizada S, Kandari JA, Wani V, Gayed I, Kumar N. Applications of endoscopy in vitreoretinal surgery. Retina. 2008;28(1):159-66.

10. Raizada S, Al Sabti K. Endoscope assisted enhanced internal search for iatrogenic retinal breaks in 20-gauge macular surgery. Br J Ophthalmol. 2012;96(7):1040. https://doi.org/10.1136/bjo.2010.199844.

11. Kaga T, Yokoyama S, Kojima T, et al. Novel endoscope-assisted vitreous surgery combined with atmospheric endoscopic technique and/or subretinal endoscopic technique for rhegmatogenous retinal detachment with grade C proliferative vitreoretinopathy. Retina. 2019;39(6):1066-75.

12. Yokoyama S, Kojima T, Mori T, et al. Clinical outcomes of endoscopeassisted vitrectomy for treatment of rhegmatogenous retinal detachment. Clin Ophthalmol. 2017;11:2003-10.

13. Rezende F, Vila N. New era in endoscopic vitreoretinal surgery. Graefes Arch Clin Exp Ophthalmol. 2019;24(10):019-04468.

14. Machemer $R$, Aaberg TM, Freeman HM, Irvine AR, Lean JS, Michels RM. An updated classification of retinal detachment with proliferative vitreoretinopathy. Am J Ophthalmol. 1991;112(2):159-65. https://doi.org/10.1016/ s0002-9394(1014)76695-76694.

15. Vila N, Rampakakis E, Rezende F. Endoscopy-assisted vitrectomy outcomes during silicone oil removal after complex retinal detachment repair. J VitreoRetinal Dis. 2019;3(6):445-51. https://doi.org/10.1177/24741 26419861850.

\section{Publisher's Note}

Springer Nature remains neutral with regard to jurisdictional claims in published maps and institutional affiliations.

Ready to submit your research? Choose BMC and benefit from

- fast, convenient online submission

- thorough peer review by experienced researchers in your field

- rapid publication on acceptance

- support for research data, including large and complex data types

- gold Open Access which fosters wider collaboration and increased citations

- maximum visibility for your research: over 100M website views per year

At BMC, research is always in progress.

Learn more biomedcentral.com/submissions 